Aim of the study: This study aims to analyze the clinical manifestations and sequelae of peripheral nerve radiation damage of two cases of cancer patients after radiotherapy at the corresponding sites in clinical practice and to summarize experiences and lesions in order to provide a reference for future tumor radiotherapy.

Material and methods: Some data of two cases of patients, such as doses of radiotherapy, clinical manifestations and damage occurrence time, were collected and examinations were conducted to define diagnosis. Afterwards, therapies and follow-up were conducted.

Results: Case 1 (rectal cancer) was diagnosed as mild left lower extremity nerve damage. After the symptomatic treatment, the disease condition was improved, and there was no tumor recurrence sign. Case 2 (breast cancer) was diagnosed as left brachial plexus damage, and left upper extremity movement function was lost completely. While the analgesic treatment was conducted, anti-tumor relevant treatments were being carried out.

Conclusions: Radiotherapy can cause different extents of radioactive nerve damage. In practice, it is necessary to constantly improve the radiotherapy technology level and actively prevent the occurrence of complications. Once symptoms appear, the diagnosis and treatment should be conducted as early as possible in order to avoid aggravating damage to cause dysfunction and cause lifetime pain to patients.

Key words: radiotherapy, radiation damage, peripheral nerve.

\section{Clinical observation of peripheral nerve injury in 2 patients with cancer after radiotherapy}

\author{
Zhao-hui Zhang ${ }^{1}$, Li Liang', Ting-zhen Jia', Shu-lan Zhang', Mo-pei Wang', \\ Li-Wen Ma', Qiang Liu' \\ 1Department of Tumor Chemotherapy and Radiation Sickness, Peking University Third \\ Hospital, Beijing, China \\ 2Institute of Radiation Medicine of Chinese Academy of Medical Science \& Peking \\ Union Medical College, China
}

\section{Introduction}

With the rapid development of tumor therapeutics, antineoplastic measures are increasing. The comprehensive application of multiple treatment methods greatly enhances tumor treatment efficiency, prolongs the survival time of patients and improves patients' life quality. Meanwhile, side effects correspondingly decrease and ease, but occur occasionally. Radiotherapy-caused damage will cause a great pain to patients, and radioactive nerve damage is one kind of radiotherapy-caused damage [1]. At present, there is still no effective treatment method, and it is an important problem in the field of radiotherapy.

Radiotherapy-caused peripheral nerve damage mainly occurs at two large positions [2]. For patients with breast cancer and head and neck tumor, radiotherapy will cause brachial plexus damage. Secondly, for patients with rectal cancer, radiotherapy after urogenital tumorectomy will cause lumbosacral plexus injury. A number of cases of radiotherapy-caused peripheral nerve damage have been reported. Johnsson [3] reported brachial plexus damage of 71 cases of patients after radiotherapy following complete breast hysterectomy, and the main clinical manifestations included upper extremity pain, weakness, sensation loss and paralysis and upper extremity paresis. After 34-year follow-up, the incidence rate was $63 \%$. Among the surviving patients, the percentage of patients with brachial plexus damage caused by radiotherapy was $89 \%$, and the percentage of patients with upper extremity paresis was $83 \%$.

\section{Aim of the study}

This study analyzes peripheral nerve radiation damage in two cases of patients with cancer (rectal cancer and breast cancer) after radiotherapy at the corresponding sites in clinical practice and summarizes experiences and lesions by a retrospective analysis in order to provide a reference for future tumor radiotherapy.

\section{Material and methods}

\section{Case 1}

Male, 57 years old. In 2005, enteroscheocele was conducted due to hemafecia to find rectal occupying lesions, and its pathologic diagnosis was rectal carcinoma. In the local hospital, radical resection of rectal carcinoma was carried out, and the clinical stage was stage III C. After operation, the adjuvant radiotherapy was carried out: three-field pelvic radiotherapy, $10 \mathrm{MV}$ X-ray and 30 Gy cumulative dose for 21 days. As the radiation damage of the patient's local skin was severe and radiation proctitis appeared, the radiotherapy was 
suspended for 2 weeks and then continued: pelvic anterior and posterior fields, 10 MV X-ray and 12 Gy dose. After one month following completion of radiotherapy, the patient gradually presented left lower extremity numbness, without pain or fever. Also, he was not specially treated. Myodynamic examination showed that bilateral muscle forces were of grade $\mathrm{V}$ and pain sense slightly reduced. Also, physiological responses existed, and no pathological reaction was reflected. Afterwards, he received chemotherapy. As a result, left lower extremity numbness was aggravated, and he occasionally suffered from pain. Repeated intermittent drug therapies of neurotrophic drugs such as vitamin $B_{1}$, cobamamide and mecobalamine and hormone drugs were conducted. In addition, special hyperbaric chamber treatment was carried out.

\section{Case 2}

Female, 54 years old. In 2004, she underwent modified radical mastectomy of breast cancer in our hospital. Postoperative pathologic diagnosis was invasive ductal carcinoma, and tumorous clinical stage was stage II B (T2N1M0). Immunohistochemistry showed three negative expressions (ER, PR and Cerb- $B_{2}$ expressions were negative). After the operation, routine chemotherapy was conducted [FEC (5-fluorouracil, epidoxorubicin and cyclophosphamide) regimen for 4 cycles and sequential paclitaxel chemotherapy for 2 cycles]. Two years postoperatively, left supraclavicular lymph node metastasis occurred. After radiotherapy, the lesion was completely relieved. Four years postoperatively, left supraclavicular lymph node cancer recurred. While chemotherapy was conducted, local radiotherapy was carried out: left chest wall radiotherapy (DT 50 Gy), 50 Gy cumulative dose of radiotherapy at the left supraclavicular lymph node area and 20 Gy local additional dose. As a result, the skin presented radiation damage of grade I. At one month after radiotherapy, she felt left upper extremity pain and numbness, and the pain and numbness were aggravated gradually. Auxiliary examinations included nuclear magnetic resonance examination and multiple ultrasound examinations. Therefore, vitamin $B_{12}$ and $B_{1}$ and hormone drugs were administered for treatment. At the same time, tramadol hydrochloride was administered, but the analgesic effect was unsatisfactory. So, gabapentin $(0.1 \times 48 / 0.3 \mathrm{tid}) /$ oxycodone \& acetaminophen tablet/neurotropin (4 IU $\times 30 \times 2$ bottles/8 IU bid), fentanyl and celecoxib $(0.2 \times 18 / 0.2$ bid $)$ were additionally administered.

\section{Results}

\section{Case 1}

In multiple electrophysiological examination results of double lower limb nerves, both lower limbs presented radioactive damage of tibial nerve (sensory fiber was severe), and left lower extremity damage was apparent. Also, there was neurogenic damage (left abductor pollicis brevis). After vitamin $B_{1}$, cobamamide, mecobalamine and hyperbaric chamber therapies were conducted, symptoms of the patient were alleviated somewhat, but he was not cured. At the end of treatment, the symptoms were aggravated somewhat. Therefore, he received therapies of neurotrophic drugs and analgesic drugs by fits. No tumor recurrence evidence was found.

\section{Case 2}

Nuclear magnetic resonance examination results of brachial plexus: left brachial plexus signal heightened and thickened, and peripheral edema occurred. Also, ipsilateral neck root muscle edema was apparent (Fig. 1). It was considered as radiation damage. At this time, ultrasound examination did not show local tumor invasion. After the symptomatic treatment was conducted (see method section), the pain of the patient were alleviated. After half a year, left hand finger flexion changed. After one year, left hand movement function was lost, and a superficial sensation existed. Multiple ultrasound examinations showed that the left supraclavicular fossa lymph node did not obviously swell, but the brachial plexus was thickened. The thickest brachial plexus reached $0.4 \times 0.3 \mathrm{~cm}$. Afterwards, therapies of analgesic drugs were always conducted by fits. At present, while toxic and anesthetic drugs are administered for analgesic treatment, antitumor relevant therapies are still being conducted.

\section{Discussion}

Clinical manifestations of radioactive nerve damage are diverse, mainly including allodynia and movement disorder of the affected limb. Besides the clinical manifestations, it is still necessary to conduct auxiliary examinations such as nerve electrophysiology or nuclear magnetic resonance for a definite diagnosis. At present, nuclear magnetic resonance is the optimal means for the diagnosis of radioactive nerve damage [4]. Regular postoperative re-examination is one of the extremely important measures for timely finding whether tumors reoccur and radioactive nerve lesions occur, and it is useful for early intervention and treatment. In this report, case 1 was definitely diagnosed by the neural electrophysiological examination. For case 2 , it was impossible to judge the reason for nerve damage only by the neural electrophysiological examination. Only by combining nuclear magnetic resonance and ultrasound examinations of the local brachial plexus is it possible to diagnose brachial plexus as radiation damage rather than tumor invasion, which provides a basis for further treatment of the patient.

As for the latency of brachial plexus damage after radiotherapy, some authors have reported that it ranged from 6 months to 20 years, and the mean was 1.5 years [5]. In the two cases reported in this study, case 1 presented left lower extremity pain and numbness at about 1 month after adjuvant radiotherapy following resection of rectal cancer. Case 2 presented left upper extremity pain and numbness at about one month after the local radiotherapy due to supraclavicular lymph node recurrence following the modified radical mastectomy of breast cancer. The left upper extremity gradually presented a mild paralysis to complete paralysis within 2 years, which was possibly associated with the total dose absorbed by the local tissues. The occurrence time of peripheral nerve damage of the two cases of patients after radiotherapy was earlier than that reported in the literature.

For the threshold dose of causing radioactive nerve damage, it is different in different reports. In the report of John- 

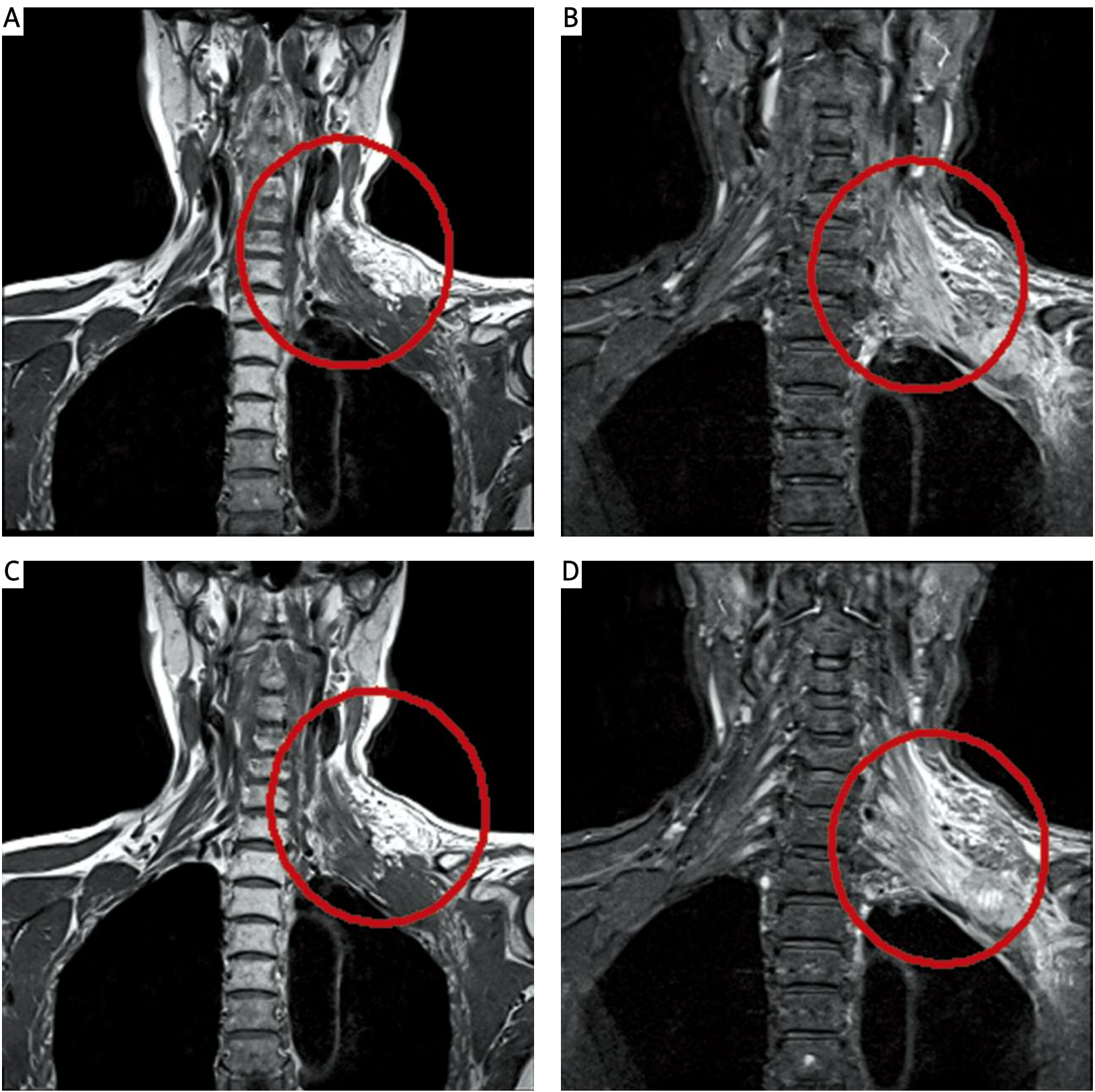

Fig. 1. Brachial plexus MR: left supraclavicular lymph nodes became narrowed after radiotherapy, but the integral signal of the left brachial plexus nerve increased compared to the right, the nerve became thick, courseware around the nerve of the brachial plexus was wide with soft tissue edematous changes [3T Magnetom Trim (Siemens): A, C: SE T1W, TR/TE $=708 / 11 \mathrm{~ms}$, thickness of layer $=2.5 \mathrm{~mm}$; B, C: STIR TR/TE $=6400 / 34, \mathrm{TI}=220 \mathrm{~ms}$, thickness of layer $=3 \mathrm{~mm}$

sson [3], in the case of 57 Gy total irradiation dose, 63 Gy effective biological dose and 3.5 Gy average single dose, the incidence rate after 34-year follow-up was 63\%. The research results of many authors [6-8] suggested that the effective biological dose rose with the increase of total dose. Therefore, the incidence rate of radioactive nerve damage of patients with breast cancer induced by radiotherapy obviously increases. Jacek et al. [9] found that when the total dose rose from 43.5 Gy to 60 Gy and a single dose rose from 2.2 Gy to 4.58 Gy, the incidence rate increased from $1.7 \%$ to $73 \%$. If a single dose was controlled in the range of 2.2 Gy to 2.5 Gy and the total dose was controlled in the range of $34 \mathrm{~Gy}$ to $40 \mathrm{~Gy}$, the incidence rate was less than $1 \%$. If the effective biological dose was more than $55 \mathrm{~Gy}$, the incidence rate obviously increased. In a word, the irradiation dose is a main factor of influencing the incidence rate of this disease. In this study, the local radiotherapy dose of the two cases of patients with breast cancer is $70 \mathrm{~Gy}$, and it is obviously far more than the above reported dose. The radiotherapy dose of rectal cancer is $42 \mathrm{~Gy}$, within the reported range. Therefore, it is very important to correctly prepare treatment planning and accurately calculate the expected dose before radiotherapy.

A majority of authors $[10,11]$ think that the main causes of radiation inducing peripheral nerve damage lie in the damage of the nerve sheath, Schwann cell, nerve microvessel and neurofilament protein caused by the direct effect of ray and ray-induced extrusion and stretching of perineural fibrosis to nerves, neural ischemia and hypoxia caused by perineural 
vascular injury and secondary injuries caused by chronic radiation inflammation, and telangiectatic lesions can also occur [12]. So, some authors propose that the exact terminology of radioactive nerve damage should be "radioactive neuropathy" [13].

Therapies of radioactive peripheral nerve damage depend on the clinical manifestations and the time and the extent of neuropathy of patients. In the early stage, hormones (adrenal cortical hormones) and neurotrophic drugs [14] (vitamin B, mecobalamin and ganglioside drugs) can be administered. Some authors also obtained certain treatment efficiency by applying anticoagulant therapy [15]. A hyperbaric chamber is also one measure for treating nerve damage [16], but it can achieve higher treatment efficiency only by combining with other auxiliary treatment measures. In addition, hyaluronic acid and some traditional Chinese medicines can play a certain remission role $[17,18]$. In the later stage, if no tumor reoccurs, it is still feasible to carry out lysis therapy [19-21]. In this study, as the clinical manifestations of case 1 were mild, application of neurotrophic drugs could relieve his symptoms. The damage extent of case 2 was severe; early application of neurotrophic drugs could not relieve or prevent disease progression. In the later stage, complete movement disorder occurred and pain was obvious. Therefore, it was necessary to administer opioids for pain relief and additionally conduct adjuvant therapy of anti-anxiety drugs.

In conclusion, medical staff should strengthen the improvement of radiotherapy technology and actively prevent the occurrence of complications. Once symptoms appear, it is necessary to conduct diagnosis as early as possible and actively treat the peripheral nerve with neurotrophic drugs in order to avoid aggravating damage to cause dysfunction and cause lifetime pain to patients.

\section{References}

1. Ko K, Sung DH, Kang MJ, et al. Clinical, electrophysiological findings in adult patients with non-traumatic plexopathies. Ann Rehabil Med 2011; 35: 807-15.

2. Dropcho EJ. Neurotoxicity of radiation therapy. Neurol Clin 2010; 28 : 217-34.

3. Johansson S, Svensson H, Larsson LG, Denekamp J. Brachial plexopathy after postoperative radiotherapy of breast cancer patientsa long-term follow up. Acta Oncol 2000; 39: 373-82.

4. Iyer VR, Sanghvi DA, Merchant N. Malignant brachial plexopathy: A pictorial essay of MRI findings. Indian J Radiol Imaging 2010; 20: 274-8.

5. Fathers E, Thrush D, Huson SM, Norman A. Radiation-induced brachia plexopathy in women treated for carcinoma of the breast. Clin Rehabil 2002; 16: 160-5.

6. Bajrovic A, Rades D, Fehlauer F, Tribius S, Hoeller U, Rudat V, Jung H, Alberti $W$. Is there a life-long risk of brachial plexopathy after radiotherapy of supraclavicular lymph nodes in breast cancer patients? Radiother Oncol 2004; 71: 297-301.

7. Fairchild AM, Weir LM, Mates D, et al. Locoregional radiation for high risk breast cancer-results of short fractionation. Breast Cancer Research Treatment 2000; 64: 35-8.

8. Livsey JE, Magee B, Stewart AL, Swindell R. Auxillary recurrence following conservative surgery and radiotherapy in early breast cancer. Clin Oncol 2000; 12: 309-14.

9. Gałecki J, Hicer-Grzenkowicz J, Grudzień-Kowalska M, Michalska T, Załucki W. Radiation induced brachial plexopathy and hypofractionated regimens in adjuvant irradiation of patients with breast can cer-a review. Acta Oncologica 2006; 45: 280-4.
10. Olsen NK. Pfeitier P. Johannsen L, Schrøder H, Rose C. Radiation-induced brachial plexopathy: neurological follow-up in 161 recurrence-free breast cancer patients. Int J Radiat Oncol Biol Phys 1993; 26: 43-9.

11. Lin YS, Jen YM, Lin JC. Radiation-related cranial nerve palsy in patients with nasopharyngeal carcinoma. Cancer 2002; 95: 404-9.

12. Kumar AJ, Leeds NE, Fuller GN, Van Tassel P, Maor MH, Sawaya RE, Levin VA. Malignant gliomas: MR imaging spectrum of radiation therapy- and chemotherapy-induced necrosis of the brain after treatment. Radiology 2000; 217: 377-84.

13. Pradat PF, Maisonobe T, Psimaras D, Lenglet T, Porcher R, Lefaix JL, Delanian S. Radiation-induced neuropathies: Collateral damage of improved cancer prognosis. Rev Neurol (Paris) 2012; 168: 939-50.

14. Eblan MJ, Corradetti MN, Lukens JN, et al. Brachial plexopathy in apical non-small cell lung cancer treated with definitive radiation: dosimetric analysis and clinical implications. Int J Radiat Oncol Biol Phys 2013; 85: 175-81.

15. Chen AM, Hall WH, Li J, Beckett L, Farwell DG, Lau DH, Purdy JA. Brachial plexus-associated neuropathy after high-dose radiation therapy for head-and-neck cancer. Int J Radiat Oncol Biol Phys 2012; 84: 165-9.

16. Amini A, Yang J, Williamson R, et al. Dose constraints to prevent radiation-induced brachial plexopathy in patients treated for lung cancer. Int J Radiat Oncol Biol Phys 2012; 82: e391-8.

17. Avila EK, Goenka A, Fontenla S. Bilateral phrenic nerve dysfunction: a late complication of mantle radiation. J Neurooncol 2011; 103: 393-5.

18. Desai KR, Nemcek AA Jr. latrogenic brachial plexopathy due to improper positioning during radiofrequency ablation. Semin Intervent Radiol 2011; 28: 167-70.

19. Hijal T, Al Hamad AA, Niazi T, Sultanem K, Bahoric B, Vuong T, Muanza T. Hypofractionated radiotherapy and adjuvant chemotherapy do not increase radiation-induced dermatitis in breast cancer patients. Curr Oncol 2010; 17: 22-7.

20. Platteaux N, Dirix P, Hermans R, Nuyts S. Brachial plexopathy after chemoradiotherapy for head and neck squamous cell carcinoma. Strahlenther Onkol 2010; 186: 517-20.

21. Jaeckle KA. Neurologic manifestations of neoplastic and radiationinduced plexopathies. Semin Neurol 2010; 30: 254-62.

\section{Address for correspondence}

\section{Zhao-hui Zhang}

Department of Tumor Chemotherapy and Radiation Sickness

Peking University Third Hospital,

No. 49 Huayuan Road Haidian District

Beijing 100191, China

tel. 86-010-82265231

e-mail: zhangzh603@126.com

Submitted: 28.07 .2012

Accepted: $\quad 03.10 .2012$ 